\title{
An investigation of the pattern formed by soil transfer when clothing fabrics are placed on soil using visual examination and image processing analysis
}

\author{
Kathleen R. Murray ${ }^{1,2 *}$, Robert W. Fitzpatrick ${ }^{1,2}$, Ralph Bottrill ${ }^{2,3}$ and Hilton Kobus ${ }^{2,4}$ \\ ${ }^{1}$ School of Biological Sciences, The University of Adelaide, Adelaide, Australia \\ ${ }^{2}$ Centre for Australian Forensic Soil Science, CSIRO Land and water, Urrbrae, Australia \\ ${ }^{3}$ Mineral Resources Tasmania, Rosny Park, Australia \\ ${ }^{4}$ Forensic Science Research Centre, Flinders University, Bedford Park, Australia
}

\begin{abstract}
A series of soil transference experiments led to the development of two methods to identify and interpret trace soil patterns on clothing fabric. Instigated by an unsolved murder, this paper developed previous field experiments to visually interpret trace soil patterns on a simulated clothed victim placed on soil during a crime. Soil transfer patterns were easily identified using direct visual analysis. Image processing software was used to analyse digital photographs and provided objective, standardised and comprehensive numerical data. This object-oriented classification of all trace soil objects transferred ( $\geq 2$ pixels) allowed directionality and abundance of transferred soil to be determined and additionally Munsell soil colour classification could be made.
\end{abstract}

To gain greater understanding of how the physical transference of soil on a victim's clothing might indicate the circumstances of an attack, 400 soil transfer experiments investigated two methods of placing weighted fabric on a wet or dry soil surface. The methodology has now been applied in other case work.

\section{Introduction}

Forensic soil evidence has the ability to help police solve crime by providing valuable associative physical evidence in any circumstance where it is found [1-9]. However, a void in forensic knowledge was discovered during a homicide investigation in Western Australia. During the trial in 2012, which was before a judge only, the judge concluded that the mineralogy data from the brick particles on the victim's bra and the bricks from her front driveway suggested she was initially attacked in her front yard and not where her body was buried [10-14].

However, at the time of the trial, no scientific research had been undertaken to interpret trace soil evidence patterns on common clothing fabric types. Therefore, the circumstances of the attack could not be ascertained from this soil evidence alone $[12,14]$. This murder remains unsolved.

Recent soil transfer experiments (STEs), both in the laboratory and field, documented distinct differences in trace soil patterns when a simulated clothed human body is either dragged or placed upon a soil surface [15-17]. Up to 20 different soil types and five common clothing fabrics were tested to better understand the influence of a specific soil transfer method, soil type, fabric type and surface irregularities/ appendages (clothing seams, plastic buttons and metal buckles) on resulting trace soil patterns on fabric. An important outcome of this work was the publication of results from testing different soil transference methods relevant to soil evidence on clothing (mainly from a bra) from the unsolved homicide from Western Australia [10-14].

Using the same 20 soil types and four fabrics (cotton, polyester- cotton, nylon and polar fleece (polyester brushed on both sides)), 400 STEs were conducted to advance scientific knowledge of forensic soil science.

\section{Materials and methods}

\section{Soil samples}

Twenty (20) natural and anthropogenic soils [18] (otherwise known as Technosol $[19,20]$ or human-altered or human-transported (HAHT) [21]), originated from three locations: the University of Tasmania (UTas) Research Farm at Richmond Rd, Cambridge, Pinnacle Rd on Mount Wellington and the Royal Tasmanian Botanical Gardens (RTBG), Lower Domain Rd, Hobart, Tasmania, Australia (Figure 1) [22]. Table 1 provides a summary of the soil morphology and classifications of these soil samples. Full descriptions of each soil is provided in Murray et al. [22].

\section{Experimental design}

Placing experiments: The method devised by Murray et al. $[15,16,22]$ to simulate a lifeless or incapacitated clothed human body dragged across a soil surface, was adapted to test two transfer methods mimicking the placement of a clothed body on a soil surface. A glass

Correspondence to: Kathleen R. Murray, School of Biological Sciences, The University of Adelaide, Adelaide, Australia, Kathleen.Murray@adelaide.edu.au

Key words: clothing fabric, forensic, image-processing, placing, soil, transference

Received: January 28, 2017; Accepted: February 15, 2017; Published: February 17, 2017 


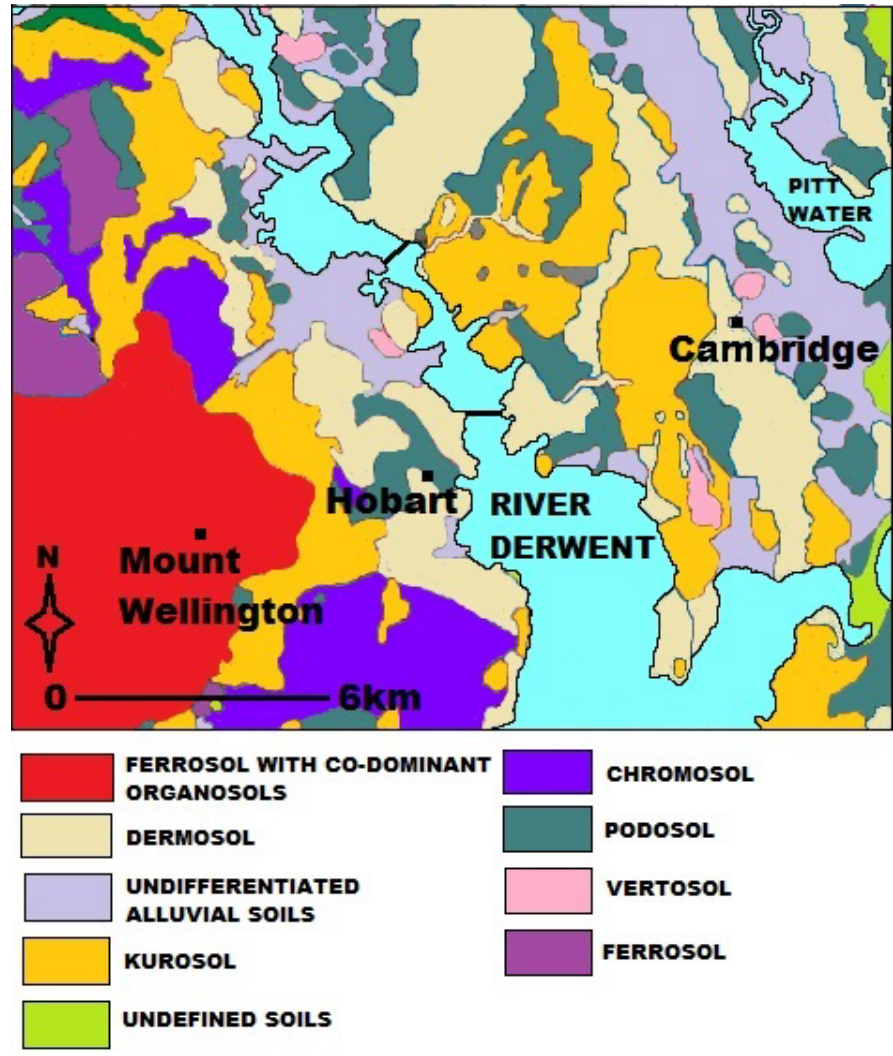

Figure 1. Soil map shows the three soil site areas of Cambridge, Hobart and Mount Wellington, Tasmania [23]; provided by Murray et al. [22].
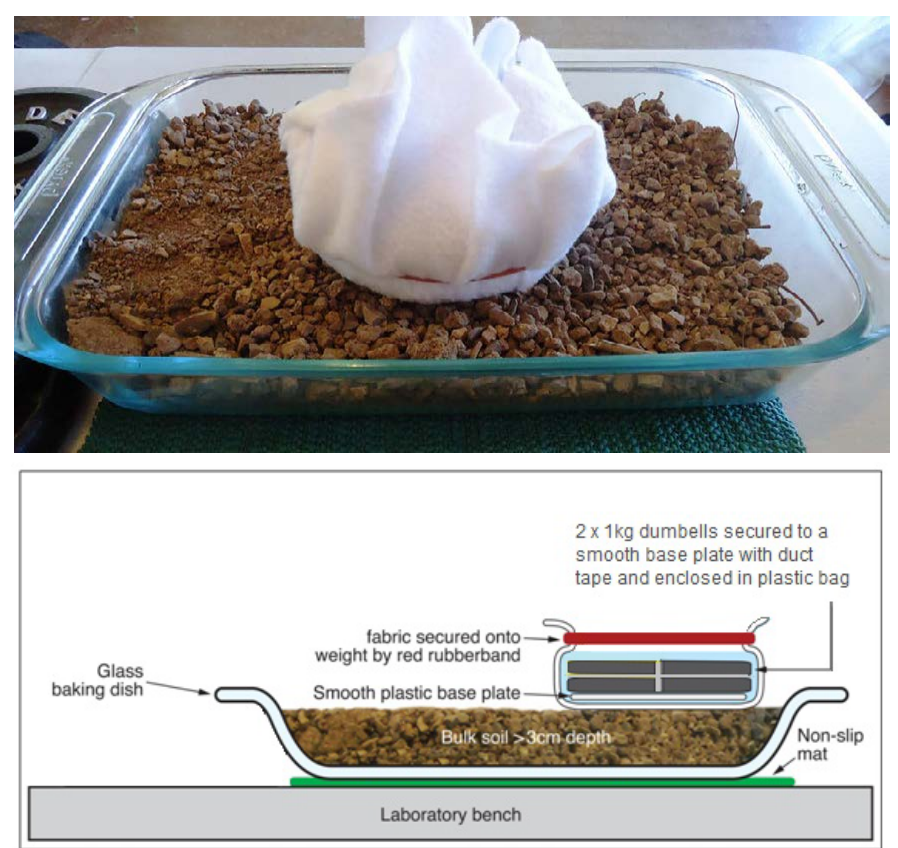

Figure 2. Photograph (above) and cross-section of a weighted fabric square placed on soil material using placement method 1, during a two minute count Soil Transference Experiment (STE). The glass dish containing bulk soil was placed on a nonslip mat to prevent movement during each STE.

Pyrex $3 \mathrm{qt} / 2.8 \mathrm{~L}$ dish was filled to $>3 \mathrm{~cm}$ with bulk soil and placed on a non-slip mat. Experiments were conducted using both wet and dry soil. To mimic clothing seams, the $30 \mathrm{~cm}^{2}$ fabric squares were sewn down the middle with a raised seam. Using the same method as Murray et al.
[22], half of the cotton squares had a $1 \mathrm{~cm}$ diameter plastic button sewn onto them. White coloured fabric was chosen to enable easy detection of trace soil transferred in minute amounts.

Using placement method 1, a fabric square was secured to a $2 \mathrm{~kg}$ weight by a strong rubber-band. this simulated firm-fitting clothing worn by a human victim. In order to achieve reproducible and consistent results, the weighted fabric was placed on wet or dry soil in a glass baking dish and timed for 2 minutes (Figure 2). Great care was taken to minimise any movement of the fabric across the soil surface. The weighted fabric was then carefully lifted up and placed on a clean surface, in order to immediately photograph the soil transference pattern. The attached weight not only stabilised soil transfer patterns, but kept the surface to be photographed and photomicrographed flat during analysis.

Placement method 2 simulated a clothed body wearing loosefitting clothing. Randomly folded non-weighted fabric was placed on a wet or dry soil surface (Figure 3). Immediately, the $2 \mathrm{~kg}$ weight was gently placed on top and the placement timed for 2 minutes. The weight would then carefully be lifted off and the fabric square gently lifted and spread flat and soil-side-up on a clean bench for photographing with a digital camera.

These digital photographs were then uploaded to a computer with image processing software which had been manually programmed to convert its default RGB colour system to the Munsell soil colour system preferred by forensic soil scientists in the field [25-27]. Full details regarding the use of image processing software to identify the Munsell soil colour of trace soil evidence is discussed in Murray et al. [15-17]. This research focused primarily on the visual interpretation of trace soil patterns on clothing to identify the circumstances befalling a victim during an attack.

Numerical data on each trace soil pattern provided a scientific and objective analysis of trace soil patterns that could be used to confirm patterns identified by human eye. Image software was also programmed to gather data on the quantity and directionality of soil objects transferred. Directionality was used to better understand the soil transfer method and was graphed using quick and easily compared rose diagrams with GEOrient version 9.5.0 [28]. Due to limitations in the manual programming of this software, soil objects $<100 \mu \mathrm{m}$ were disregarded from analysis [15-17,22].

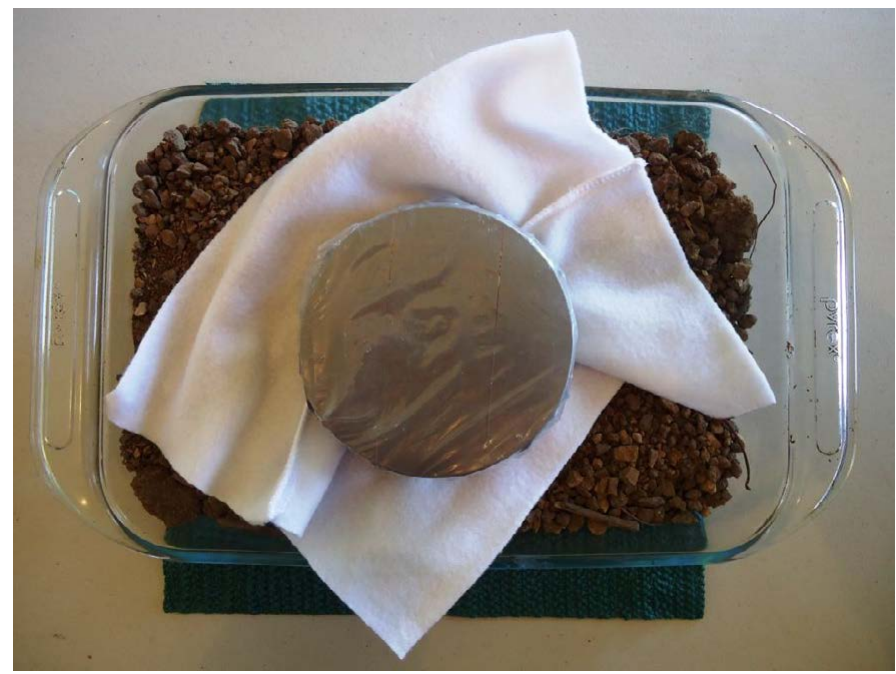

Figure 3. Using placement method 2, loosely-folded fabric is first placed on a soil surface before a $2 \mathrm{~kg}$ weight is placed on top and the 2 minute count commences. 
Murray KR (2017) An investigation of the pattern formed by soil transfer when clothing fabrics are placed on soil using visual examination and image processing analysis

Table 1. Soil morphology and Australian Soil Classification of soil materials [18] and approximate corresponding classifications for World Reference Base for Soil resources class [19] provided by Murray et al. [22]

\begin{tabular}{|c|c|c|c|c|c|c|}
\hline Locality (depth $\mathrm{cm}$ ) & $\begin{array}{c}\text { Centre for } \\
\text { Australian Forensic } \\
\text { Soil Science } \\
\text { (CAFSS) code }\end{array}$ & \begin{tabular}{|} 
Munsell' ${ }^{1}$ Soil \\
Colour fine $<2 \mathbf{~ m m}$ \\
(wet) (dry)
\end{tabular} & Soil type $^{2}$ & Brief description & $\begin{array}{l}\text { The Australian soil } \\
\text { classification }\end{array}$ & $\begin{array}{l}\text { The world reference base } \\
\text { for soil resources (WRB) }\end{array}$ \\
\hline $\begin{array}{c}\text { Site 1: UTas farm } \\
\text { horizon } 1(0-10 \mathrm{~cm})\end{array}$ & 110.1 .1 & $\begin{array}{l}\text { Very dark brown } \\
\text { 10YR } 2 / 2 \text { Dark } \\
\text { grayish brown } \\
\text { 10YR 4/2 }\end{array}$ & $\begin{array}{c}\text { Duplex soil with } \\
\text { nonrestricted clayey subsoil }\end{array}$ & $\begin{array}{l}\text { Gravel }(50 \%) \text {; clayey sand, } \\
\text { water repellent, } 6.92 \% \\
\text { Carbon }(\mathrm{C}) .\end{array}$ & $\begin{array}{c}\text { Humose, Mesotrophic, } \\
\text { Brown Chromosol; medium, } \\
\text { moderately gravelly, loamy, } \\
\text { clayey, moderate }\end{array}$ & $\begin{array}{l}\text { Haplic, Luvisol (Clayic, } \\
\text { Cutanic) }\end{array}$ \\
\hline $\begin{array}{c}\text { Site 1: UTas farm } \\
\text { horizon } 2(15-30 \mathrm{~cm})\end{array}$ & 110.1 .2 & $\begin{array}{c}\text { Very dark brown } \\
\text { 10YR 2/2 Brown } \\
\text { 10YR 5/3 }\end{array}$ & As above & $\begin{array}{c}\text { Gravel }(45 \%) \text {; sandy loam, } \\
\text { water repellent, } 2.46 \% \\
\text { Carbon }(\mathrm{C})\end{array}$ & As above & As above \\
\hline $\begin{array}{c}\text { Site 1: UTas farm } \\
\text { horizon } 3(40-60 \mathrm{~cm})\end{array}$ & 110.1 .3 & $\begin{array}{l}\text { Strong brown } \\
\text { 7.5YR 4/6 Strong } \\
\text { brown } 7.5 \text { YR } 5 / 6\end{array}$ & As above & $\begin{array}{l}\text { Gravel }(90 \%) \text {; heavy clay, } \\
\text { non-water repellent, } 0.92 \% \\
\text { Carbon }(\mathrm{C})\end{array}$ & As above & As above \\
\hline $\begin{array}{c}\text { Site 1: UTas farm } \\
\text { horizon } 4(60-80 \mathrm{~cm})\end{array}$ & 110.1 .4 & \begin{tabular}{|c|} 
Strong brown \\
7.5YR 5/6 Reddish \\
yellow 7.5YR 6/6
\end{tabular} & As above & $\begin{array}{c}\text { Gravel }(90 \%) \text {; medium- } \\
\text { heavy clay, non-water } \\
\text { repellent, } 0.26 \% \text { Carbon }(\mathrm{C}) .\end{array}$ & As above & As above \\
\hline $\begin{array}{l}\text { Site 1: UTas farm } \\
\text { horizon } 5(80-100 \\
\mathrm{cm})\end{array}$ & 110.1 .5 & \begin{tabular}{|c|} 
Dark brown \\
7.5YR 3/4 Reddish \\
yellow 7.5 YR $6 / 6$
\end{tabular} & As above & $\begin{array}{l}\text { Gravel }(40 \%) \text {; clayey sand, } \\
\text { non-water repellent, } 0.19 \% \\
\text { Carbon (C). }\end{array}$ & As above & As above \\
\hline $\begin{array}{c}\text { Site 2: UTas farm } \\
\text { horizon } 1(0-17 \mathrm{~cm})\end{array}$ & 110.2 .1 & $\begin{array}{l}\text { Very dark brown } \\
\text { 10YR } 2 / 2 \text { Grayish } \\
\text { brown } 10 \text { YR } 5 / 2\end{array}$ & $\begin{array}{l}\text { Duplex soil with restricted } \\
\text { sodic clayey subsoil }\end{array}$ & $\begin{array}{l}\text { Gravel }(35 \%) \text {; sandy loam, } \\
\text { non-water repellent, } 2.61 \% \\
\text { Carbon }(\mathrm{C})\end{array}$ & $\begin{array}{c}\text { Vertic, Mottled-Mesonatric, } \\
\text { Brown Sodosol; medium, } \\
\text { moderately gravelly, loamy, } \\
\text { clayey, deep }\end{array}$ & $\begin{array}{l}\text { Vertic, Abruptic Solonetz } \\
\text { (Albic, Hypernatric) }\end{array}$ \\
\hline $\begin{array}{c}\text { Site 2: UTas farm } \\
\text { horizon } 2(17-34 \mathrm{~cm})\end{array}$ & 110.2 .2 & $\begin{array}{c}\text { Brown 10YR 4/3 } \\
\text { Pale brown 10YR } \\
6 / 3\end{array}$ & As above & $\begin{array}{c}\text { Gravel }(95 \%) \text {; medium- } \\
\text { heavy clay, non-water } \\
\text { repellent, } 1.14 \% \text { Carbon }(\mathrm{C}) .\end{array}$ & As above & As above \\
\hline $\begin{array}{c}\text { Site 3: UTas farm } \\
\text { horizon } 1(0-10 \mathrm{~cm})\end{array}$ & 110.3 .1 & $\begin{array}{l}\text { Very dark gray } \\
\text { 10YR 3/1 Dark } \\
\text { grayish brown } \\
\text { 10YR 4/2 }\end{array}$ & $\begin{array}{l}\text { Poorly structured cracking } \\
\text { clay }\end{array}$ & $\begin{array}{c}\text { Gravel }(90 \%) \text {; medium } \\
\text { clay, water repellent, } 2.37 \% \\
\text { Carbon }(\mathrm{C}) .\end{array}$ & $\begin{array}{l}\text { Endocalcareous, Massive, } \\
\text { Brown Vertosol; very } \\
\text { gravelly, fine, very fine, deep }\end{array}$ & $\begin{array}{l}\text { Calsic, Vertisol (Gilgaic, } \\
\text { Gleyic) }\end{array}$ \\
\hline $\begin{array}{c}\text { Site 3: UTas farm } \\
\text { horizon } 2(20-40 \mathrm{~cm})\end{array}$ & 110.3 .2 & $\begin{array}{c}\text { Yellowish brown } \\
\text { 10YR 5/4 Grayish } \\
\text { brown 10YR 4/2 }\end{array}$ & As above & $\begin{array}{c}\text { Gravel }(97 \%) \text {; heavy clay, } \\
\text { non-water repellent, } 0.84 \% \\
\text { Carbon }(\mathrm{C})\end{array}$ & As above & As above \\
\hline $\begin{array}{c}\text { Site 3: UTas farm } \\
\text { horizon } 3(40-70 \mathrm{~cm})\end{array}$ & 110.3 .3 & $\begin{array}{c}\text { Light yellowish } \\
\text { brown 10YR } 6 / 4 \\
\text { Light gray 10YR } \\
7 / 2\end{array}$ & As above & $\begin{array}{l}\text { Gravel }(90 \%) \text {; medium } \\
\text { clay, non-water repellent, } \\
3.54 \% \text { Carbon }(C) .\end{array}$ & As above & As above \\
\hline $\begin{array}{l}\text { Site 3: UTas farm } \\
\text { horizon } 4 \\
(70-110 \mathrm{~cm})\end{array}$ & 110.3 .4 & $\begin{array}{l}\text { Yellowish brown } \\
\text { 10YR 5/4 Brown } \\
\text { 10YR 5/3 }\end{array}$ & As above & $\begin{array}{l}\text { Gravel }(90 \%) \text {; heavy clay, } \\
\text { non-water repellent, } 0.44 \% \\
\text { Carbon }(\mathrm{C})\end{array}$ & As above & As above \\
\hline $\begin{array}{c}\text { Site 4: Mount } \\
\text { Wellington horizon } 1 \\
(0-10 \mathrm{~cm})\end{array}$ & 110.4 .1 & $\begin{array}{c}\text { Very dark brown } \\
\text { 10YR } 2 / 2 \text { Very } \\
\text { dark grayish brown } \\
\text { 10YR } 3 / 2\end{array}$ & $\begin{array}{l}\text { Well structured clayey soil } \\
\text { with boulders }\end{array}$ & $\begin{array}{l}\text { Gravel }(70 \%) \text {; sandy clay } \\
\text { loam, water repellent, } \\
12.8 \% \text { Carbon }(C) .\end{array}$ & $\begin{array}{c}\text { Humose-Mottled, Placic, } \\
\text { Brown Kandosol; medium, } \\
\text { moderately gravelly, loamy, } \\
\text { clayey, deep }\end{array}$ & $\begin{array}{l}\text { Xanthic, Ferretic, Ferralsol } \\
\text { (Clayic, Colluvic) }\end{array}$ \\
\hline $\begin{array}{c}\text { Site 4: Mount } \\
\text { Wellington horizon } 3 \\
\quad(40-60 \mathrm{~cm})\end{array}$ & 110.4 .3 & $\begin{array}{l}\text { Strong brown } \\
7.5 Y R 4 / 6 \\
\text { Brownish yellow } \\
\text { 10YR 6/6 }\end{array}$ & As above & $\begin{array}{l}\text { Gravel }(70 \%) \text {; sandy clay } \\
\text { loam, non-water repellent, } \\
0.96 \% \text { Carbon }(\mathrm{C})\end{array}$ & As above & As above \\
\hline $\begin{array}{c}\text { Site } 4 \text { : Mount } \\
\text { Wellington horizon } 5 \\
(110-140 \mathrm{~cm})\end{array}$ & 110.4 .5 & $\begin{array}{l}\text { Strong brown } \\
\text { 7.5YR 4/6 Strong } \\
\text { brown } 7.5 \text { YR } 5 / 6\end{array}$ & As above & $\begin{array}{l}\text { Gravel }(75 \%) \text {; sandy clay } \\
\text { loam, non-water repellent, } \\
0.34 \% \text { Carbon }(\mathrm{C})\end{array}$ & As above & As above \\
\hline $\begin{array}{l}\text { Site 5: rose garden } \\
\text { path }(0-10 \mathrm{~cm})\end{array}$ & 110.5 .1 & $\begin{array}{c}\text { Dark brown } \\
\text { 7.5YR 3/2 Brown } \\
\text { 7.5YR 5/2 }\end{array}$ & $\begin{array}{l}\text { Anthropogenic gravelly } \\
\text { sandy loam soil }\end{array}$ & $\begin{array}{c}\text { Gravel ( } 90 \% \text {; arkosic } \\
\text { sandstone and andesitic-to- } \\
\text { weathered mafic igneous } \\
\text { rock) loamy sand, water } \\
\text { repellent, } 0.7 \% \text { Carbon }(\mathrm{C}) .\end{array}$ & $\begin{array}{l}\text { Spolic anthroposol, very } \\
\text { gravelly, sandy, very shallow }\end{array}$ & ${ }^{3}$ Spolic technosol (Densic) \\
\hline $\begin{array}{c}\text { Site 6: brick } \\
\text { fragments }(0-2 \mathrm{~cm})\end{array}$ & 110.6 .2 & $\begin{array}{c}\text { Red 2.5YR 5/8 } \\
\text { Light red } 2.5 Y R \\
7 / 8\end{array}$ & $\begin{array}{l}\text { Anthropogenic brick } \\
\text { fragment-rich soil }\end{array}$ & $\begin{array}{l}\text { Gravel }(90 \%) \text {, sandy, water } \\
\text { repellent, weathered brick } \\
\text { fragments }(0.5-4 \mathrm{~cm}), 1.2 \% \mathrm{C} .\end{array}$ & $\begin{array}{l}\text { Urbic anthroposol, very } \\
\text { gravelly, sandy, very shallow }\end{array}$ & $\begin{array}{l}{ }^{3} \text { Urbic Ekranic technosol } \\
\text { (Transportic) }\end{array}$ \\
\hline $\begin{array}{l}\text { Site 7: rose garden } \\
\quad \text { bed }(0-10 \mathrm{~cm})\end{array}$ & 110.7 .1 & \begin{tabular}{|c} 
Black 10YR 2/1 \\
Black 7.5YR 2.5/1
\end{tabular} & $\begin{array}{l}\text { Anthropogenic, organic-rich } \\
\text { sandy loam soil }\end{array}$ & $\begin{array}{c}\text { Gravel }(15 \% \text { coarse river } \\
\text { sand), loamy sand }(25 \%) \text {, } \\
\text { water repellent, } 30 \% \text { fine } \\
\text { compost, } 30 \% \text { composted } \\
\text { pine bark, } 14 \% \text { C. }\end{array}$ & $\begin{array}{l}\text { Hortic anthroposol non- } \\
\text { gravelly, sandy, shallow }\end{array}$ & $\begin{array}{l}{ }^{4} \text { Hortic anthrosol } \\
\text { (Escalic) }\end{array}$ \\
\hline $\begin{array}{l}\text { Site 8: Japanese } \\
\text { garden bed } \\
(0-10 \mathrm{~cm})\end{array}$ & 110.8 .1 & $\begin{array}{l}\text { Dark reddish gray } \\
\text { 2.5YR 3/1 Reddish } \\
\text { gray } 2.5 \mathrm{YR} 6 / 1\end{array}$ & $\begin{array}{l}\text { Anthropogenic, quartz-rich, } \\
\text { gravelly, sandy soil }\end{array}$ & $\begin{array}{c}\text { Gravel ( } 90 \%: \sim 80 \% \\
\text { rounded quartz, } 10 \% \\
\text { sub-rounded to angular } \\
\text { dolerite;5\% ironstone), } \\
\text { loamy sand, water repellent, } \\
3 \% \mathrm{C} \text {. }\end{array}$ & $\begin{array}{l}\text { Spolic anthroposol, very } \\
\text { gravelly, sandy, very shallow }\end{array}$ & $\begin{array}{c}{ }^{3} \text { Spolic technosol } \\
\text { (Grossartefactic, Transportic) }\end{array}$ \\
\hline
\end{tabular}


Murray KR (2017) An investigation of the pattern formed by soil transfer when clothing fabrics are placed on soil using visual examination and image processing analysis

\begin{tabular}{|c|c|c|c|c|c|c|}
\hline $\begin{array}{l}\text { Site 9: south eastern } \\
\text { boundary horizon } 1 \\
\qquad(5-0 \mathrm{~cm})\end{array}$ & 110.9 .1 & \begin{tabular}{|c|} 
Leaves not \\
analysed for \\
Munsell soil colour \\
by naked eye
\end{tabular} & Natural organic-rich soil & $\begin{array}{l}\text { Undecomposed Leaves } \\
(60 \%) \text { and decomposed } \\
(40 \%)\end{array}$ & $\begin{array}{l}\text { Humose, mesotrophic, } \\
\text { Brown Dermosol, non- } \\
\text { gravelly, sandy, deep }\end{array}$ & Eutric Cambisol (Humic) \\
\hline $\begin{array}{l}\text { Site 9: south eastern } \\
\text { boundary horizon } 2 \\
(0-10 \mathrm{~cm})\end{array}$ & 110.9 .2 & $\begin{array}{c}\text { Very dark brown } \\
\text { 10YR } 2 / 2 \text { Very } \\
\text { dark brown } 7.5 \mathrm{YR} \\
2.5 / 2\end{array}$ & Natural loamy soil & $\begin{array}{l}\text { Gravel }(2 \%) \text {, loamy sand, } \\
\text { water repellent, } 23 \% \mathrm{C} \text {. }\end{array}$ & As above & As above \\
\hline
\end{tabular}

Where:

${ }^{1}$ Munsell soil colour [29]: measured on the fine earth fraction $(<2 \mathrm{~mm})$.

${ }^{2}$ Special-purpose technical soil classification system [24], which uses plain English and places strong emphasis on being either an anthropogenic soil or natural soil, the soil texture (e.g. gravelly, sandy, sandy loam) and the presence of high quantities of organic carbon ( $>10 \%$; organic-rich).

${ }^{3}$ Classification of technosols [19]: Connotation: soils dominated or strongly influenced by human-made material; from Greek technikos, skilfully made. They contain a significant amount of artefacts.

${ }^{4}$ Classification of Anthroposol [19]: Connotation: soils with prominent characteristics that result from human activities; from Greek anthropos, human being (e.g. such as addition of organic material and cultivation)

${ }^{5}$ Classification of natural soils: Connotation: soils with substantial soil formation such as Dermosols [18] or Cambisols [19].

Table 2. Trace soil patterns identified on fabric using 'placing' as the transfer method.

\begin{tabular}{|c|c|c|c|c|c|}
\hline & Soil transfer pattern & Symbol used & Figure & Location on fabric & Contributing soil characteristic \\
\hline 1 & Speckling of soil on fabric & red circle & $\begin{array}{l}\text { Figure } 4 \\
\text { Figure } 5 \\
\text { Figure } 8\end{array}$ & $\begin{array}{l}\text { sporadically where fabric had } \\
\text { made contact with soil surface }\end{array}$ & $\begin{array}{c}\text { Marginally greater quantity of } \\
\text { wet soil objects transferred } \\
(96 \% \text { consistency) than dry }(90 \%)\end{array}$ \\
\hline 2 & $\begin{array}{c}\text { Fold and crease marks } \\
\text { on draped cloth (Method 2) }\end{array}$ & green circle & $\begin{array}{l}\text { Figure } 4 \\
\text { Figure } 5\end{array}$ & $\begin{array}{l}\text { delineating folds or creases } \\
\text { on loosely draped cloth }\end{array}$ & $\begin{array}{l}100 \% \text { consistency in all soils } \\
\text { both dry and wet }\end{array}$ \\
\hline 3 & Soiled water stains & blue circle & $\begin{array}{l}\text { Figure } 5 \\
\text { Figure } 6\end{array}$ & $\begin{array}{l}\text { sporadically where fabric had } \\
\text { made contact with soil surface }\end{array}$ & $\begin{array}{c}\text { wet soil } \\
(45 \% \text { consistency })\end{array}$ \\
\hline 4 & 'Dusted' plastic buttons & orange circle & Figure 7 & $\begin{array}{l}\text { buttons sewn onto cotton fabric } \\
\text { (no other fabrics had buttons) }\end{array}$ & $\begin{array}{c}\text { very dry soil } \\
(55 \% \text { consistency) }\end{array}$ \\
\hline 5 & $\begin{array}{l}\text { Muddy clumps on wet } \\
\text { shiny plastic buttons }\end{array}$ & yellow circle & Figure 7 & $\begin{array}{l}\text { buttons sewn onto cotton } \\
\text { fabric }\end{array}$ & $\begin{array}{c}\text { wet soil } \\
(30 \% \text { consistency) }\end{array}$ \\
\hline 6 & $\begin{array}{l}\text { Minimal amount of soil } \\
\text { accumulated on raised } \\
\text { surfaces }\end{array}$ & - & Figure 6 & raised middle seams & $\begin{array}{l}\text { Marginally greater quantity of } \\
\text { wet soil objects transferred } \\
(19 \% \text { consistency) than dry }(6 \%)\end{array}$ \\
\hline
\end{tabular}

\section{Results and discussion}

\section{Trace soil patterns recorded on fabric using the soil transfer} method of 'placing'

When a weighted fabric square was simply placed on soil, six transfer patterns were routinely identified using all soils tested (Table 2 and Figures 4-7). As hypothesised, previously documented soil transfer patterns that occurred when weighted fabric was dragged across a soil surface, were not seen in any of these soil transfer experiments [1517,22]. The soil patterns not seen included soil 'trails' and elongated soil particles either embedded in fabric or aligned parallel with the direction of movement. Also missing was an accumulation of soil in front of buttons and raised seams, damage to fabric and scratches on buttons.

Trace soil patterns transferred to fabric depended primarily on soil moisture content, soil mineralogy (e.g. smectite) and clay fraction $(<2$ um) content. Wet soil tended to transfer a higher abundance of trace soil particles to fabric than when soil was dry. There was one exception to this, soils with a sandy clay loam texture, which is discussed in greater detail in section 3.5 below. Clothing fabric type had no observable effect on resulting trace soil patterns.

\section{Directionality}

Using directional numerical data provided by image processing software, rose diagrams illustrated the directionality of wet and dry soil particles ( $\geq 2$ pixels diameter) transferred onto clothing fabric (Figure 9). This methodology provided objective support to visual observations.

By mapping the directionality of thousands of soil particles down to $100 \mu \mathrm{m}$ diameter, rose diagrams consistently created a simple yet definitive pictorial record of soil transferred onto fabric during placing experiments. Each Rose diagram only took minutes to create.

In each of the soils tested in placing STEs, there was no obvious movement of soil seen across the fabric; as witnessed in dragging STEs from soil 'trails' [15-17,22]. Minimal to negligible soil was transferred to the weighted fabric; compared to the transfer method of dragging. This resulted in several instances when there was insufficient data to create a rose diagram or the directional data for a particular STE was so limited, it affected the rose diagram's value. When dry soil was used, dry soil particles tended to remain as individual soil objects and not clump into aggregates. This lack of soil clumping or directional soil movement also helps explain the scattered random patterns depicted in each rose diagram.

Dry hard soils, such as soils with massive structures found at UTas Farm, produced minimal soil transference patterns in placing experiments. This was also the case in the dry gravel-rich soil of the RTBG site 8 Japanese garden composed of $90 \%$ white gravel. Natural soil site 9 surface horizon 1 of undecomposed leaves, with negligible mineral soil content, transferred minimal trace soil to fabric.

Rose diagrams recorded random and scattered trace soil patterns with no obvious directionality that was seen when the same four types of fabric squares were dragged across a wet or dry soil surface [22]. The strong directionality identified by image processing software from dragging experiments was consistently seen throughout all twenty soils; regardless of fabric used or each soil type's differing mineralogy, grain size or amount of fine clay-sized soil particles. These initial experiments suggest that rose diagrams using image processing directional data 
Murray KR (2017) An investigation of the pattern formed by soil transfer when clothing fabrics are placed on soil using visual examination and image processing analysis

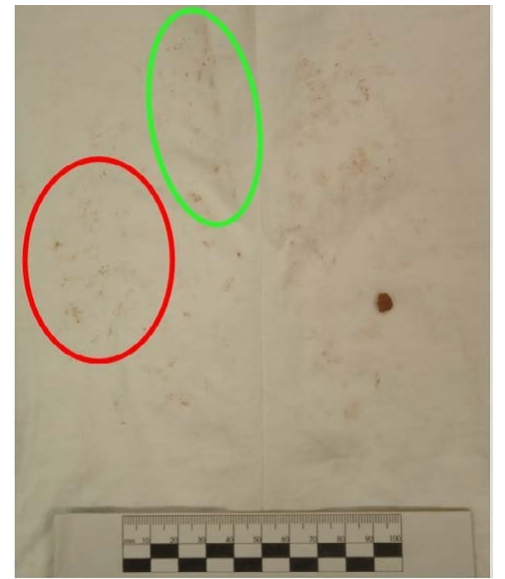

(A)DRY Site 6 brick fragments RTBG (cotton)

Figure 4(A). Trace soil patterns resulting from transfer method 2 that involved placing loosely draped weighted fabric for 2 minutes on a soil bed containing dry soil. Fabric tested: cotton. The difference soil moisture can make on resulting trace soil patterns is demonstrated by comparing the same fabric type placed on the same soil type; with the only difference being soil moisture content.

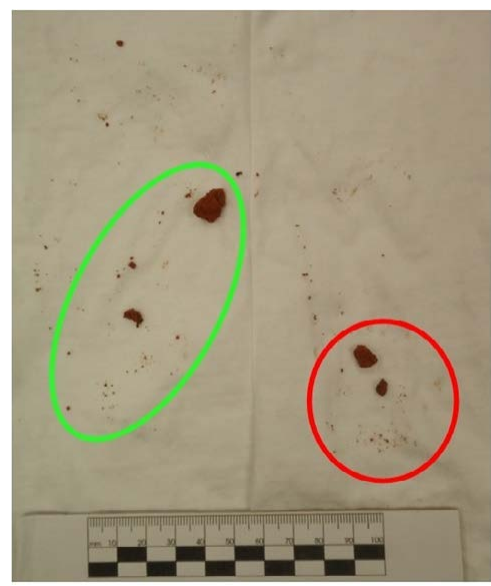

(B) WET Site 6 brick fragments (cotton)

Figure 4(B). Trace soil patterns resulting from transfer method 2 that involved placing loosely draped weighted fabric for 2 minutes on a soil bed containing wet soil. Fabric tested: cotton.

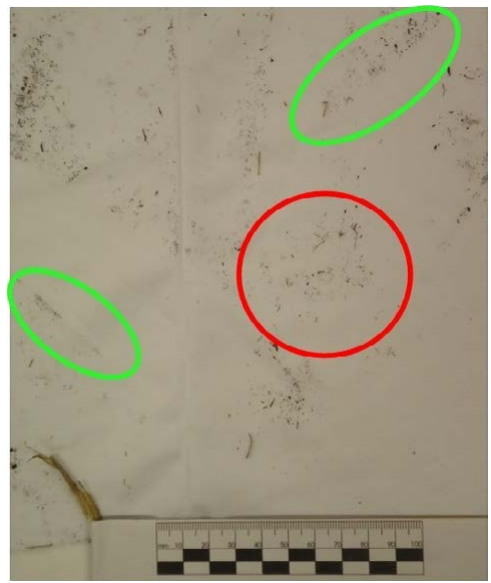

(C) DRY Site 7 rose garden bed RTBG (nylon)

Figure 4(C). Trace soil patterns resulting from transfer method 2 that involved placing loosely draped weighted fabric for 2 minutes on a soil bed containing dry soil. Fabric tested: nylon.

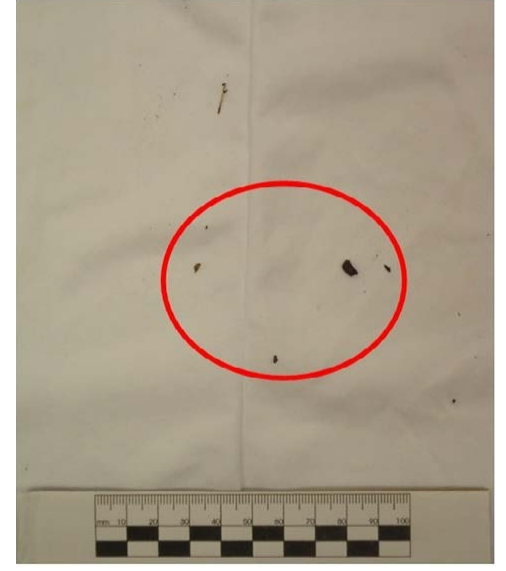

(D) WET Site 7 rose garden bed (nylon)

Figure 4(D). Trace soil patterns resulting from transfer method 2 that involved placing loosely draped weighted fabric for 2 minutes on a soil bed containing wet soil. Fabric tested: nylon.

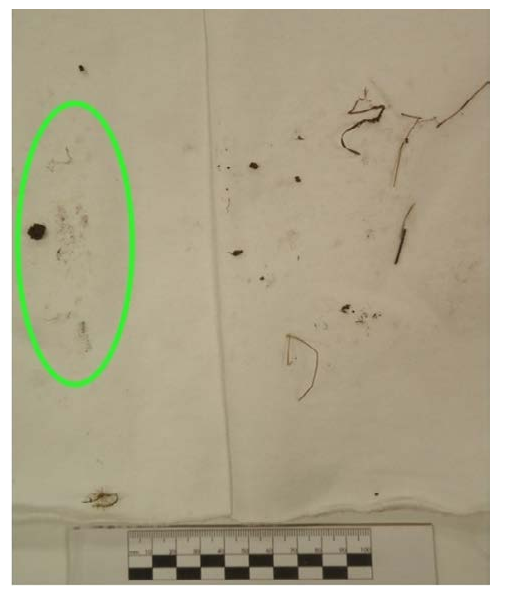

(A) DRY Site 1 surface horizon UTAS (Poly-cotton)

Figure 5(A). Trace soil patterns using transfer method 2, when loosely draped weighted fabric is placed for 2 minutes on a soil bed containing dry soil. Fabric tested: polyestercotton. When comparing Figures 5(A to D), soil moisture content appears to make a greater difference to resulting trace soil patterns than fabrics tested.

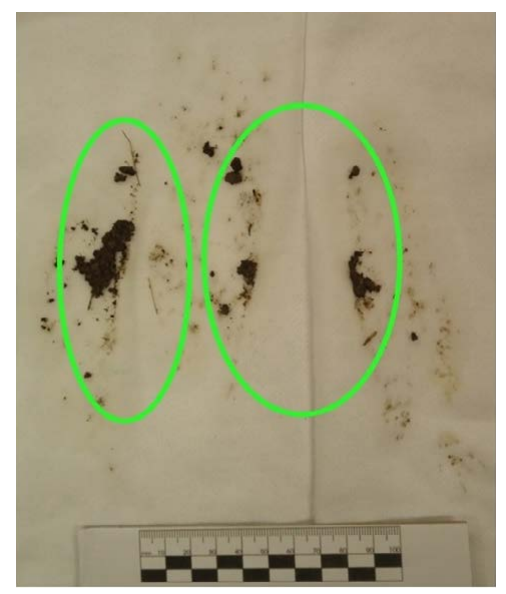

(B) WET Site 1 surface horizon (Poly-cotton)

Figure 5(B). Trace soil patterns using transfer method 2, when loosely draped weighted fabric is placed for 2 minutes on a soil bed containing wet soil. Fabric tested: polyestercotton. 
Murray KR (2017) An investigation of the pattern formed by soil transfer when clothing fabrics are placed on soil using visual examination and image processing analysis

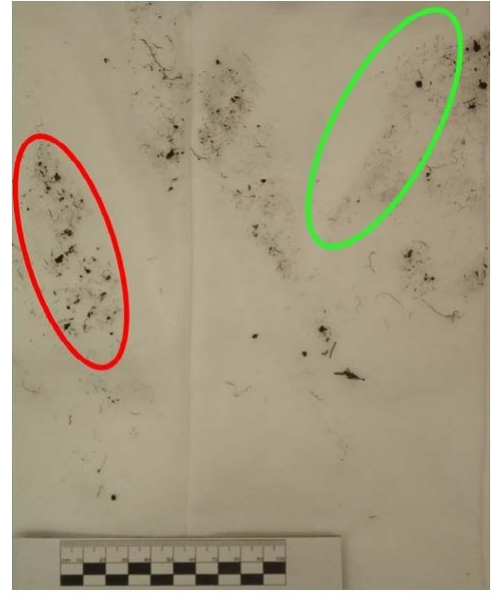

(C) DRY Site 4 horizon 1 Mt. Wellington (polar fleece)

Figure 5(C). Trace soil patterns using transfer method 2, when loosely draped weighted fabric is placed for 2 minutes on a soil bed containing dry soil. Fabric tested: polar fleece (polyester brushed both sides).

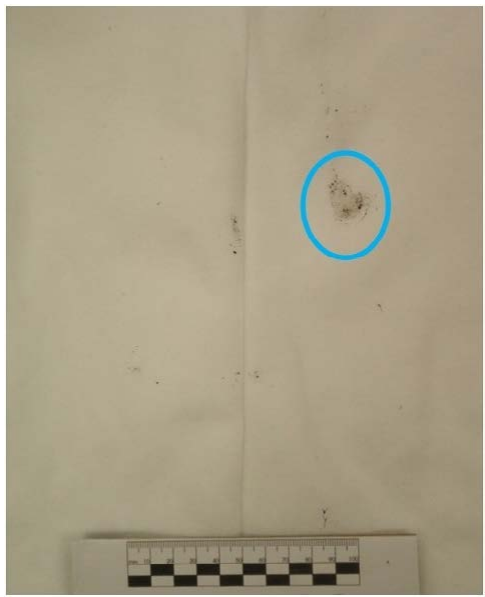

(D) WET Site 4 horizon $1 \mathrm{Mt}$. Wellington (polar fleece)

Figure 5(D). Trace soil patterns using transfer method 2, when loosely draped weighted fabric is placed for 2 minutes on a soil bed containing wet soil. Fabric tested: polar fleece (polyester brushed both sides).

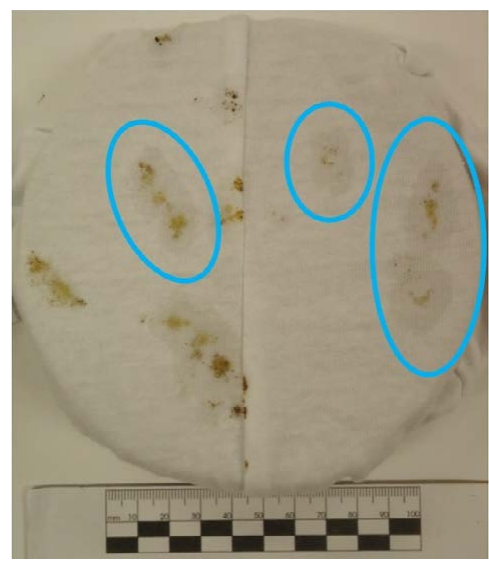

Figure 6. Wet trace soil from Site 2 horizon 1 UTas farm semi-dissolves and embeds in fabric fibres (cotton).

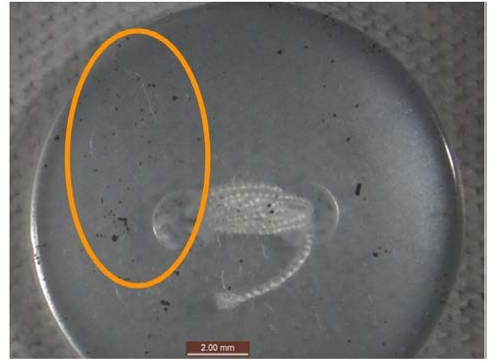

(A) DRY site 9 horizon 1 leaves, RTBG (cotton)

Figure 7(A). Photomicrograph of plastic button sewn onto cotton fabric and placed on dry soil. Button is $1 \mathrm{~cm}$ diameter. Orange circle $=$ very fine clay-sized particles 'dusted' sporadically over button.

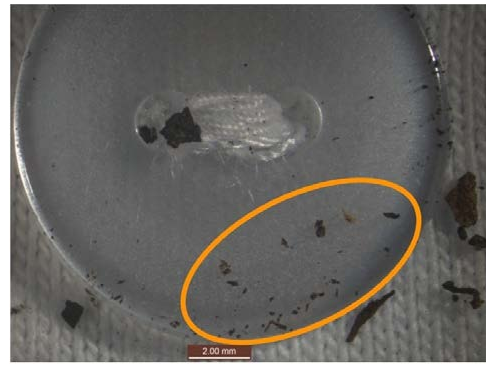

(B) DRY site 7 rose garden bed, RTBG (cotton)

Figure 7(B). Photomicrograph of plastic button sewn onto cotton fabric and placed on dry soil. Buttons $1 \mathrm{~cm}$ diameter. Orange circle $=$ very fine clay-sized particles 'dusted' sporadically over button.

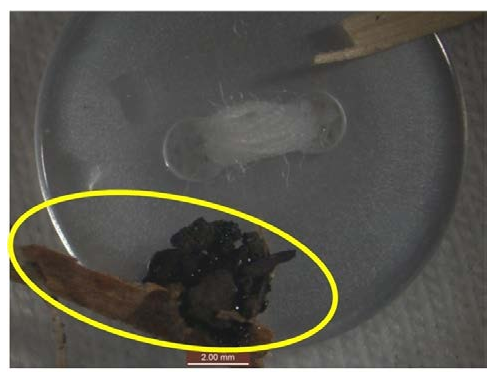

(C) WET site 7 rose garden bed (cotton)

Figure 7(C). Photomicrograph of plastic button sewn onto cotton fabric and placed on wet soil. Button $1 \mathrm{~cm}$ diameter. Yellow circle = wet clumps of soil particles persisting to button

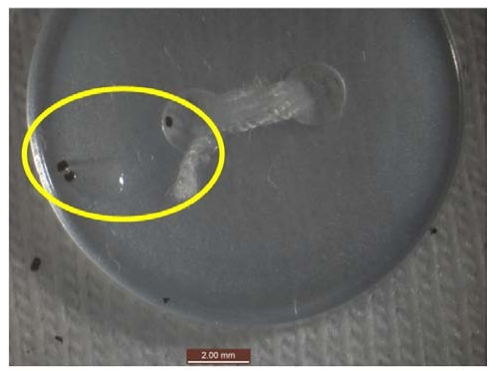

D) WET site 9 horizon 2 mineral soil (cotton)

Figure 7(D). Photomicrographs of plastic buttons sewn onto cotton fabric and placed on wet soil. Buttons $1 \mathrm{~cm}$ diameter. Yellow circle $=$ wet clumps of soil particles persisting to button. 


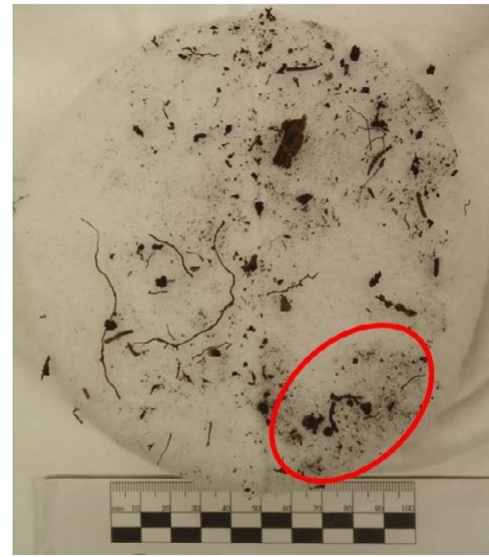

(A)DRY site 9 horizon 2 mineral soil, RTBG (polar fleece)

Figure 8(A). Polar fleece fabric speckled with soil in a dry placement on site 9 natural mineral soil, RTBG. In Figures 8 (A to B), soil moisture content had a more consistent influence on resulting trace soil patterns than did the four common fabrics tested. Red circle $=$ a patch of fabric randomly specked by soil.

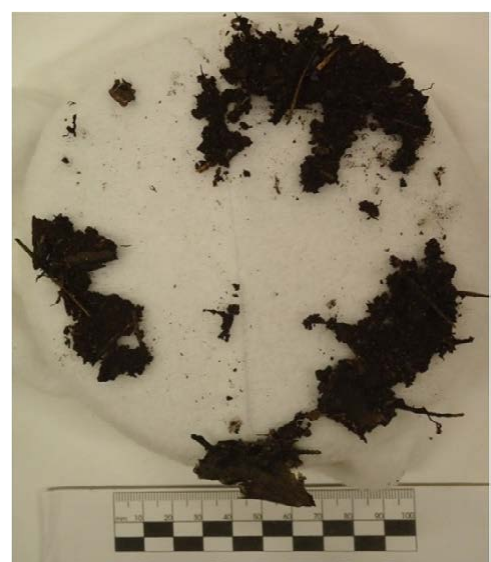

(B) WET site 9 horizon 2 mineral soil (polar fleece)

Figure 8(B). Polar fleece fabric speckled with clumps of soil in a wet placement on site 9 natural mineral soil, RTBG.

can indicate whether trace soil objects were transferred to fabric by placing or dragging a simulated clothed human body on a soil surface; regardless of the four fabric types tested.

Quantity of soil objects transferred to clothing fabric and analysed by soil type

A standardised objectivity of soil pattern analysis was achieved using quantitative graphical presentation of computer analysis of the number of digital pixels, containing either individual or aggregate soil objects (Table 3 and Figure 10). The level of precision attained when analysing trace soil patterns on clothing fabric had not previously been possible via traditional identification by naked eye alone

Soils were first grouped by location and then sub-grouped by soil moisture content. Numerical data was combined and averaged to produce graphs of aggregate and individual soil objects, transferred by one of two transfer methods of placing, from each soil sample to clothing fabric.

Soil objects covering $>0.5$ million pixels were categorised as a low quantity of soil objects transferred to fabric. Soil objects covering 0.5 million to $>1$ million pixels were categorised within a moderate

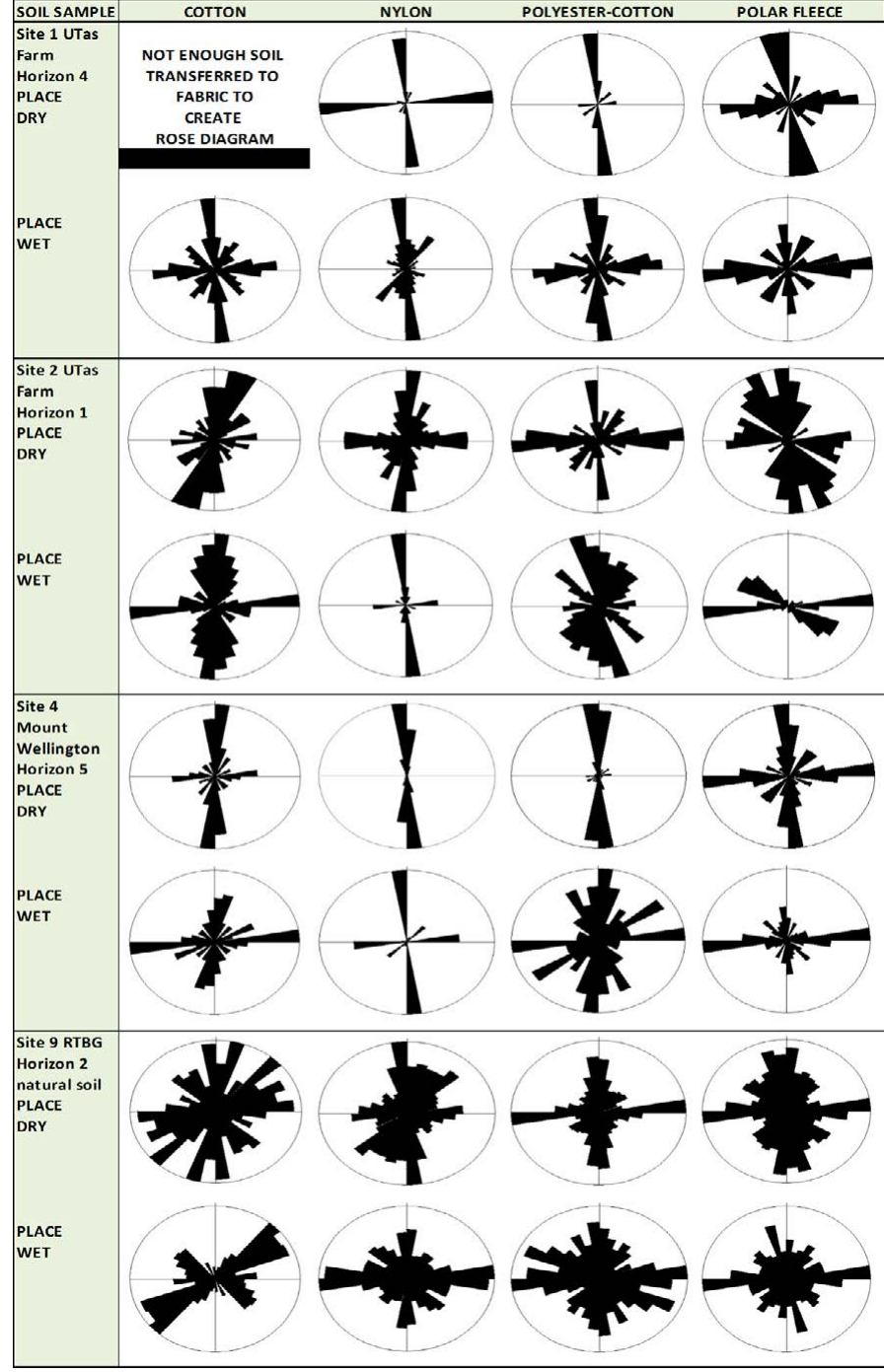

Figure 9. Rose diagrams display the directionality of wet and dry soil objects transferred onto fabric during STEs using soil from UTas Farm Site 1 horizon 4 and site 2 surface horizon, Mt. Wellington site 4 horizon 5 and RTBG site 9 mineral rich soil. A scattered and random pattern, lacking strong directionality, was produced when four fabric types were placed on a soil surface.

quantity of soil objects transferred. Soil objects covering 1 million pixels or higher were categorised as a high quantity of soil objects transferred [15-17,22].

Image processing numerical data enabled a thorough, detailed and objective interpretation of the relationships between soil types, clay minerals (smectite) and soil moisture content.

Compared to the quantity of soil objects transferred by the transfer method of dragging the same fabric types across the same 20 soils [22], the quantity of soil transferred by placing was in most cases so low as to indicate only that increased soil moisture tended to increase the quantity of soil objects transferred (Figure 11). This was the general trend across all soils tested, regardless of soil mineralogy (e.g. smectite) and clay content. The exception to this was soil with a sandy clay loam texture; namely natural soils from site 4: Mt. Wellington and Anthroposol site 5: rose garden path (Figure 10). Although site 5 was $90 \%$ gravel, the soil matrix had a sandy loam texture. 
Murray KR (2017) An investigation of the pattern formed by soil transfer when clothing fabrics are placed on soil using visual examination and image processing analysis

Table 3. Summary of soil data documented using naked eye, XRD and NDIR analysis and image processing of the 20 soil types and trace soil objects transferred to clothing fabric; as provided by Murray et al. [22].

\begin{tabular}{|c|c|c|c|c|c|c|c|c|}
\hline Locality & $\begin{array}{l}\text { Centre for } \\
\text { Australian } \\
\text { Forensic } \\
\text { Soil Science } \\
\text { (CAFSS) }\end{array}$ & $\begin{array}{c}\text { Munsell }{ }^{1} \text { soil colour } \\
<2 \mathrm{~mm} \text { fraction }\end{array}$ & $\begin{array}{l}\text { Dominant trace } \\
\text { soil }^{2} \text { Munsell } \\
\text { colour }\end{array}$ & $\begin{array}{l}\text { Ave. soil transferred } \\
\quad \text { (individual }+ \\
\text { aggregates) in pixels }\end{array}$ & & $\begin{array}{c}\text { Weight \% } \\
\text { Clay content } \\
\text { (Smectite) }\end{array}$ & $\begin{array}{c}\% \text { Organic } \\
\text { content }\end{array}$ & $\begin{array}{c}\text { \% Gravel } \\
\text { content }\end{array}$ \\
\hline (Depth cm) & code & (wet) (dry) & (wet) (dry) & $\begin{array}{l}\text { (wet) (dry) } \\
\text { High quantity } \\
\text { Moderate } \\
\text { Low }\end{array}$ & $\begin{array}{l}\text { (H) } \\
\text { (M) } \\
\text { (L) }\end{array}$ & & & \\
\hline $\begin{array}{l}\text { Site 1: UTas farm } \\
\text { horizon } 1 \\
(0-10 \mathrm{~cm})\end{array}$ & 110.1 .1 & $\begin{array}{c}\text { Very dark brown } \\
\text { 10YR } 2 / 2 \\
\text { Dark grayish brown } \\
\text { 10YR 4/2 }\end{array}$ & $\begin{array}{c}\text { Light gray 10YR } \\
7 / 2 \\
\text { Light gray } 10 \mathrm{YR} 7 / 2\end{array}$ & $\begin{array}{l}34026 \\
45481\end{array}$ & $\begin{array}{l}(\mathbf{L}) \\
(\mathrm{L})\end{array}$ & $5 \pm 1$ & 6.92 & 50 \\
\hline $\begin{array}{l}\text { Site } 1 \text { horizon } 2 \\
\quad(15-30 \mathrm{~cm})\end{array}$ & 110.1 .2 & $\begin{array}{c}\text { Very dark brown } \\
\text { 10YR 2/2 } \\
\text { Brown } 10 \text { YR } 5 / 3\end{array}$ & $\begin{array}{c}\text { Light gray 10YR } \\
7 / 2 \\
\text { Light gray } 10 \mathrm{YR} 7 / 2\end{array}$ & $\begin{array}{l}20415 \\
14212\end{array}$ & $\begin{array}{l}(\mathbf{L}) \\
(\mathrm{L})\end{array}$ & $13 \pm 2$ & 2.46 & 45 \\
\hline $\begin{array}{l}\text { Site } 1 \text { horizon } 3 \\
\quad(40-60 \mathrm{~cm})\end{array}$ & 110.1 .3 & $\begin{array}{c}\text { Strong brown } \\
\mathbf{7 . 5 Y R} \mathbf{4 / 6} \\
\text { Strong brown } 7.5 \mathrm{YR} \\
5 / 6\end{array}$ & $\begin{array}{c}\text { Light gray 10YR } \\
\mathbf{7 / 2} \\
\text { Light gray } 10 \mathrm{YR} 7 / 2\end{array}$ & $\begin{array}{c}\mathbf{7 5 0 8 7} \\
3017\end{array}$ & $\begin{array}{l}(\mathbf{L}) \\
(\mathrm{L})\end{array}$ & $56 \pm 5$ & 0.92 & 90 \\
\hline $\begin{array}{l}\text { Site } 1 \text { horizon } 4 \\
\quad(60-80 \mathrm{~cm})\end{array}$ & 110.1 .4 & $\begin{array}{c}\text { Strong brown } \\
\mathbf{7 . 5 Y R ~ 5 / 6} \\
\text { Reddish yellow } \\
7.5 \text { YR 6/6 }\end{array}$ & $\begin{array}{c}\text { Light gray } 7.5 Y R \\
7 / 2 \\
\text { Light gray } 10 Y R 7 / 2\end{array}$ & $\begin{array}{c}16783 \\
4820\end{array}$ & $\begin{array}{l}\text { (L) } \\
(\mathrm{L})\end{array}$ & $59 \pm 5$ & 0.26 & 90 \\
\hline $\begin{array}{l}\text { Site } 1 \text { horizon } 5 \\
(80-100 \mathrm{~cm})\end{array}$ & 110.1 .5 & $\begin{array}{c}\text { Dark brown } 7.5 \mathrm{YR} \\
\mathbf{3} / \mathbf{4} \\
\text { Reddish yellow } \\
\text { 7.5YR } 6 / 6\end{array}$ & $\begin{array}{c}\text { Light gray 10YR } \\
7 / 2 \\
\text { Light gray } 10 \mathrm{YR} 7 / 2\end{array}$ & $\begin{array}{c}\mathbf{2 2 9 5 9} \\
8537\end{array}$ & $\begin{array}{l}\text { (L) } \\
(\mathrm{L})\end{array}$ & $52 \pm 5$ & 0.19 & 40 \\
\hline $\begin{array}{l}\text { Site 2: UTas farm } \\
\text { horizon } 1 \\
(0-17 \mathrm{~cm})\end{array}$ & 110.2 .1 & $\begin{array}{c}\text { Very dark brown } \\
\text { 10YR 2/2 } \\
\text { Grayish brown } \\
\text { 10YR 5/2 }\end{array}$ & $\begin{array}{c}\text { Very dark brown } \\
7.5 \mathrm{YR} \mathbf{2 . 5 / 2} \\
\text { Llight gray } 10 \mathrm{YR} \\
7 / 2\end{array}$ & $\begin{array}{l}17521 \\
20775\end{array}$ & $\begin{array}{l}(\mathbf{L}) \\
(\mathrm{L})\end{array}$ & $3 \pm 1$ & 2.61 & 35 \\
\hline $\begin{array}{l}\text { Site } 2 \text { horizon } 2 \\
\quad(10-34 \mathrm{~cm})\end{array}$ & 110.2 .2 & $\begin{array}{c}\text { Brown 10YR 4/3 } \\
\text { Pale brown 10YR } \\
6 / 3\end{array}$ & $\begin{array}{c}\text { Light gray 10YR } \\
7 / 2 \\
\text { Light gray } 10 \mathrm{YR} 7 / 2\end{array}$ & $\begin{array}{c}\mathbf{6 3 4 0 8} \\
814\end{array}$ & $\begin{array}{l}\text { (L) } \\
(\mathrm{L})\end{array}$ & $5 \pm 2$ & 1.14 & 95 \\
\hline $\begin{array}{l}\text { Site 3: UTas farm } \\
\text { horizon } 1(0-10 \mathrm{~cm})\end{array}$ & 110.3 .1 & $\begin{array}{c}\text { Very dark gray } \\
\text { 10YR 3/1 } \\
\text { Dark grayish brown } \\
\text { 10YR 4/2 }\end{array}$ & $\begin{array}{c}\text { Light gray 10YR } \\
7 / 2 \\
\text { Light gray } 10 \mathrm{YR} 7 / 2\end{array}$ & $\begin{array}{c}\mathbf{5 7 4 5 7} \\
2638\end{array}$ & $\begin{array}{l}(\mathbf{L}) \\
(\mathrm{L})\end{array}$ & $41 \pm 4$ & 2.37 & 90 \\
\hline $\begin{array}{l}\text { Site } 3 \text { horizon } 2 \\
\quad(20-40 \mathrm{~cm})\end{array}$ & 110.3 .2 & $\begin{array}{c}\text { Yellowish brown } \\
\text { 10YR 5/4 } \\
\text { Grayish brown } \\
\text { 10YR } 5 / 2\end{array}$ & $\begin{array}{c}\text { Light gray 10YR } \\
7 / 2 \\
\text { Light gray } 10 \mathrm{YR} 7 / 2\end{array}$ & $\begin{array}{c}\mathbf{2 1 9 3 8} \\
382\end{array}$ & $\begin{array}{l}(\mathbf{L}) \\
(\mathrm{L})\end{array}$ & $58 \pm 5$ & 0.84 & 97 \\
\hline $\begin{array}{l}\text { Site } 3 \text { horizon } 3 \\
\quad(40-70 \mathrm{~cm})\end{array}$ & 110.3 .3 & $\begin{array}{c}\text { Light yellowish } \\
\text { brown 10YR 6/4 } \\
\text { Light gray 10YR 7/2 }\end{array}$ & $\begin{array}{c}\text { Light gray 10YR } \\
7 / 2 \\
\text { Light gray } 10 \mathrm{YR} 7 / 2\end{array}$ & $\begin{array}{c}24092 \\
864\end{array}$ & $\begin{array}{l}(\mathbf{L}) \\
(\mathrm{L})\end{array}$ & $46 \pm 4$ & 3.54 & 90 \\
\hline $\begin{array}{l}\text { Site } 3 \text { horizon } 4 \\
(70-110 \mathrm{~cm})\end{array}$ & 110.3 .4 & $\begin{array}{c}\text { Yellowish brown } \\
\text { 10YR 5/4 } \\
\text { Brown 10YR 5/3 }\end{array}$ & $\begin{array}{c}\text { Light gray 10YR } \\
7 / 2 \\
\text { Light gray } 10 \mathrm{YR} 7 / 2\end{array}$ & $\begin{array}{c}\mathbf{2 8 8 1 9} \\
1049\end{array}$ & $\begin{array}{l}(\mathbf{L}) \\
(\mathrm{L})\end{array}$ & $64 \pm 5$ & 0.44 & 90 \\
\hline $\begin{array}{l}\text { Site 4: Mount Wellington } \\
\text { horizon } 1(0-10 \mathrm{~cm})\end{array}$ & 110.4 .1 & $\begin{array}{l}\text { Very dark brown } \\
\quad \text { 10YR } \mathbf{2} / \mathbf{2} \\
\text { Very dark grayish } \\
\text { brown } 10 \mathrm{YR} 3 / 2\end{array}$ & $\begin{array}{c}\text { Light gray 10YR } \\
7 / 2 \\
\text { Light gray } 10 \mathrm{YR} 7 / 2\end{array}$ & $\begin{array}{l}30119 \\
60244\end{array}$ & $\begin{array}{l}(\mathbf{L}) \\
(\mathrm{L})\end{array}$ & $7 \pm 2$ & 12.8 & 70 \\
\hline $\begin{array}{l}\text { Site } 4 \text { horizon } 3 \\
\quad(40-60 \mathrm{~cm})\end{array}$ & 110.4 .3 & $\begin{array}{c}\text { Strong brown } \\
\text { 7.5YR 4/6 } \\
\text { Brownish yellow } \\
\text { 10YR 6/6 }\end{array}$ & $\begin{array}{c}\text { Light gray 10YR } \\
7 / 2 \\
\text { Light gray } 10 \mathrm{YR} 7 / 2\end{array}$ & $\begin{array}{c}\mathbf{7 5 7 1} \\
139787\end{array}$ & $\begin{array}{l}(\mathbf{L}) \\
(\mathrm{L})\end{array}$ & $10 \pm 2$ & 0.96 & 70 \\
\hline $\begin{array}{l}\text { Site } 4 \text { horizon } 5 \\
(110-140 \mathrm{~cm})\end{array}$ & 110.4 .5 & $\begin{array}{c}\text { Strong brown } \\
\mathbf{7 . 5 Y R} \mathbf{4} / \mathbf{6} \\
\text { Strong brown } 7.5 \mathrm{YR} \\
5 / 6\end{array}$ & $\begin{array}{c}\text { Light gray 10YR } \\
7 / 2 \\
\text { Light gray } 10 \mathrm{YR} 7 / 2\end{array}$ & $\begin{array}{l}\mathbf{2 6 4 2 3} \\
35937\end{array}$ & $\begin{array}{l}\text { (L) } \\
(\mathrm{L})\end{array}$ & $5 \pm 2$ & 0.34 & 75 \\
\hline $\begin{array}{l}\text { Site 5: RTBG rose } \\
\text { garden path } \\
(0-10 \mathrm{~cm})\end{array}$ & 110.5 .1 & $\begin{array}{c}\text { Dark brown } \\
\text { 7.5YR 3/2 } \\
\text { Brown } \\
7.5 \text { YR } 5 / 2\end{array}$ & $\begin{array}{l}\text { Light gray } \\
\text { 10YR 7/2 } \\
\text { Light gray } \\
10 Y R 7 / 2\end{array}$ & $\begin{array}{l}22671 \\
49842\end{array}$ & $\begin{array}{l}\text { (L) } \\
\text { (L) }\end{array}$ & $19 \pm 3$ & 0.70 & 90 \\
\hline
\end{tabular}


Murray KR (2017) An investigation of the pattern formed by soil transfer when clothing fabrics are placed on soil using visual examination and image processing analysis

\begin{tabular}{|c|c|c|c|c|c|c|c|c|}
\hline $\begin{array}{l}\text { Site 6: RTBG brick } \\
\text { fragments } \\
(0-2 \mathrm{~cm})\end{array}$ & 110.6 .2 & \begin{tabular}{|c|} 
Red \\
2.5YR 5/8 \\
Light red 2.5YR 7/8
\end{tabular} & \begin{tabular}{|c|} 
Very dark brown \\
$7.5 \mathrm{YR} 2.5 / 2$ \\
Light gray $10 \mathrm{YR} 7 / 2$ \\
\end{tabular} & $\begin{array}{l}100127 \\
69957\end{array}$ & $\begin{array}{l}\text { (L) } \\
\text { (L) }\end{array}$ & Trace of Mullite & 0.29 & 90 \\
\hline $\begin{array}{l}\text { Site 7: RTBG rose } \\
\text { garden bed } \\
(0-10 \mathrm{~cm})\end{array}$ & 110.7 .1 & $\begin{array}{c}\text { Black } \\
\text { 10YR 2/1 } \\
\text { Black } \\
7.5 \text { YR 2.5/1 }\end{array}$ & 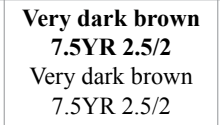 & $\begin{array}{l}\mathbf{1 5 7 5 4 2 1} \\
304925\end{array}$ & $\begin{array}{l}\text { (H) } \\
\text { (L) }\end{array}$ & $2 \pm 1$ & 14.0 & 15 \\
\hline $\begin{array}{l}\text { Site 8: RTBG Japanese } \\
\text { garden bed } \\
(0-10 \mathrm{~cm})\end{array}$ & 110.8 .1 & $\begin{array}{c}\text { Dark reddish gray } \\
\mathbf{2 . 5 Y R} \text { 3/1 } \\
\text { Reddish gray } \\
\text { 2.5YR 6/1 }\end{array}$ & $\begin{array}{c}\text { Very dark brown } \\
\mathbf{7 . 5 Y R} \mathbf{2 . 5 / 2} \\
\text { Very dark brown } \\
7.5 \text { YR } 2.5 / 2\end{array}$ & $\begin{array}{l}11690 \\
4643\end{array}$ & $\begin{array}{l}\text { (L) } \\
(\mathrm{L})\end{array}$ & $2 \pm 1$ & 3.10 & 90 \\
\hline $\begin{array}{l}\text { Site } 9 \text { : RTBG } \\
\text { SE boundary } \\
\text { horizon } 1(5-0 \mathrm{~cm} \\
\text { above surface })\end{array}$ & 110.9 .1 & $\begin{array}{l}\text { Leaves } \\
\text { leaves }\end{array}$ & $\begin{array}{c}\text { Very dark brown } \\
\mathbf{7 . 5 Y R} \mathbf{2 . 5 / 2} \\
\text { Very dark brown } \\
7.5 \text { YR } 2.5 / 2\end{array}$ & $\begin{array}{l}\mathbf{2 5 5 6 3} \\
16127\end{array}$ & $\begin{array}{l}\text { (L) } \\
(\mathrm{L})\end{array}$ & No XRD analysis & No NDIR analysis & Leaves \\
\hline $\begin{array}{l}\text { Site } 9 \text { horizon } 2 \\
\quad(0-10 \mathrm{~cm})\end{array}$ & 110.9 .2 & $\begin{array}{l}\text { Very dark brown } \\
\text { 10YR } \mathbf{2} / \mathbf{2} \\
\text { Very dark brown } \\
7.5 \text { YR } 2.5 / 2\end{array}$ & $\begin{array}{c}\text { Very dark brown } \\
\mathbf{7 . 5 Y R} \mathbf{2 . 5 / 2} \\
\text { Very dark brown } \\
7.5 \text { YR } 2.5 / 2\end{array}$ & $\begin{array}{r}\mathbf{1 5 6 5 9 9 3} \\
160684\end{array}$ & $\begin{array}{l}\text { (H) } \\
\text { (L) }\end{array}$ & $20 \pm 3$ & 22.8 & 2 \\
\hline
\end{tabular}

Where: ${ }^{1}$ Munsell Soil Colour [21]: measured by naked eye on the fine earth fraction $(<2 \mathrm{~mm})$.

${ }^{2}$ Munsell Soil Colour measured by image processing software from two digital photographs of trace soil on fabric.

${ }^{3}$ Average of two STEs tested using either wet or dry soil sample.

${ }^{4} \mathrm{XRD}$ analysis of \% weight clay (Smectite) content in homogenous bulk soil sample.

${ }^{5}$ NDIR analysis of organic content of homogenous bulk soil sample.

${ }^{6}$ Estimate by naked eye alone using method of McDonald and Isbell [30].

\section{Quantity of soil transferred to fabric (no. of pixels)}

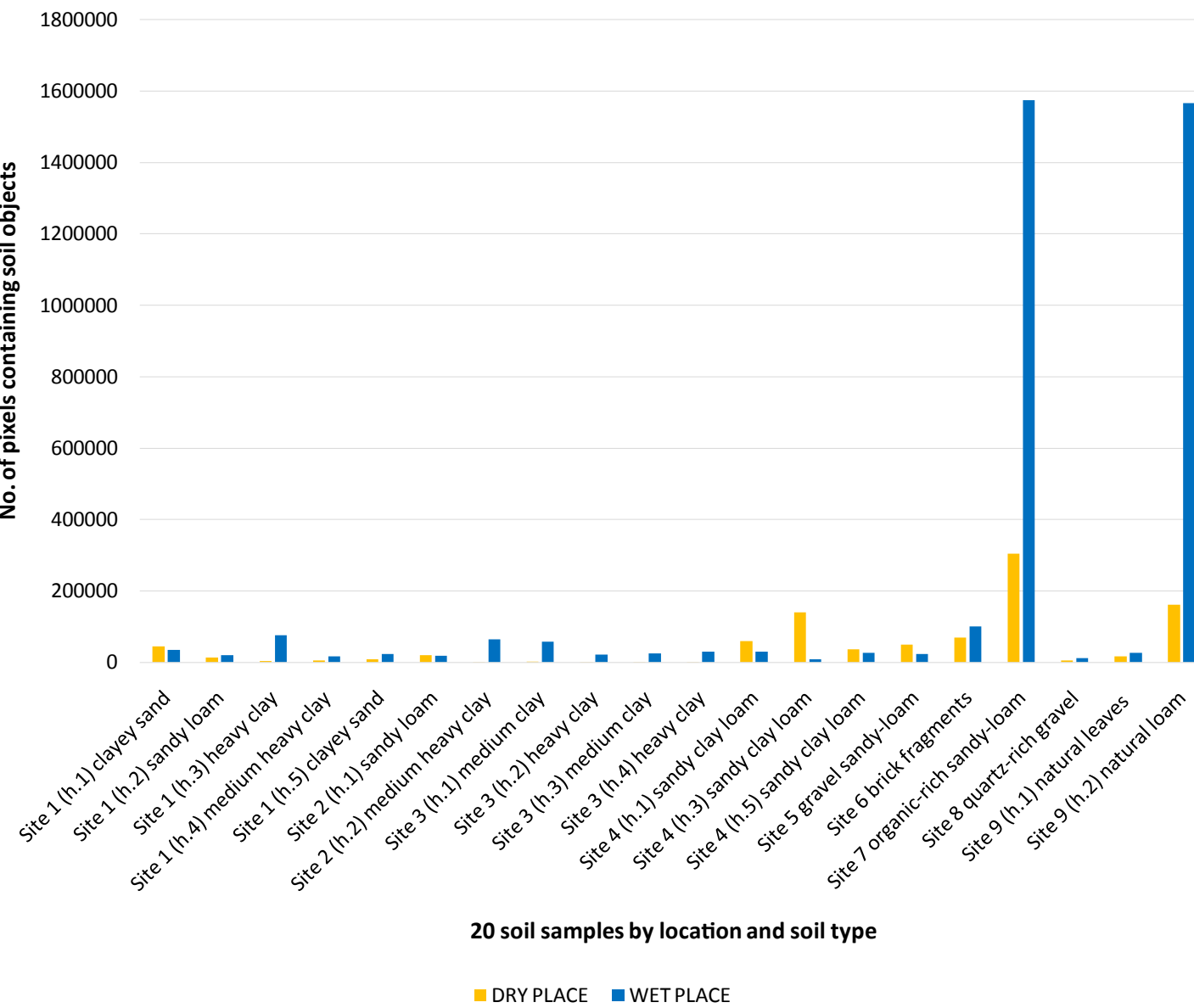

Figure 10. Quantity of soil transferred to fabric by placing weighted fabric on a soil surface; analysed by the total number of pixels in digital photographs that contained aggregate or individual soil objects. Image processing numerical data was subdivided first by location and then by soil moisture content $[15,16]$. 


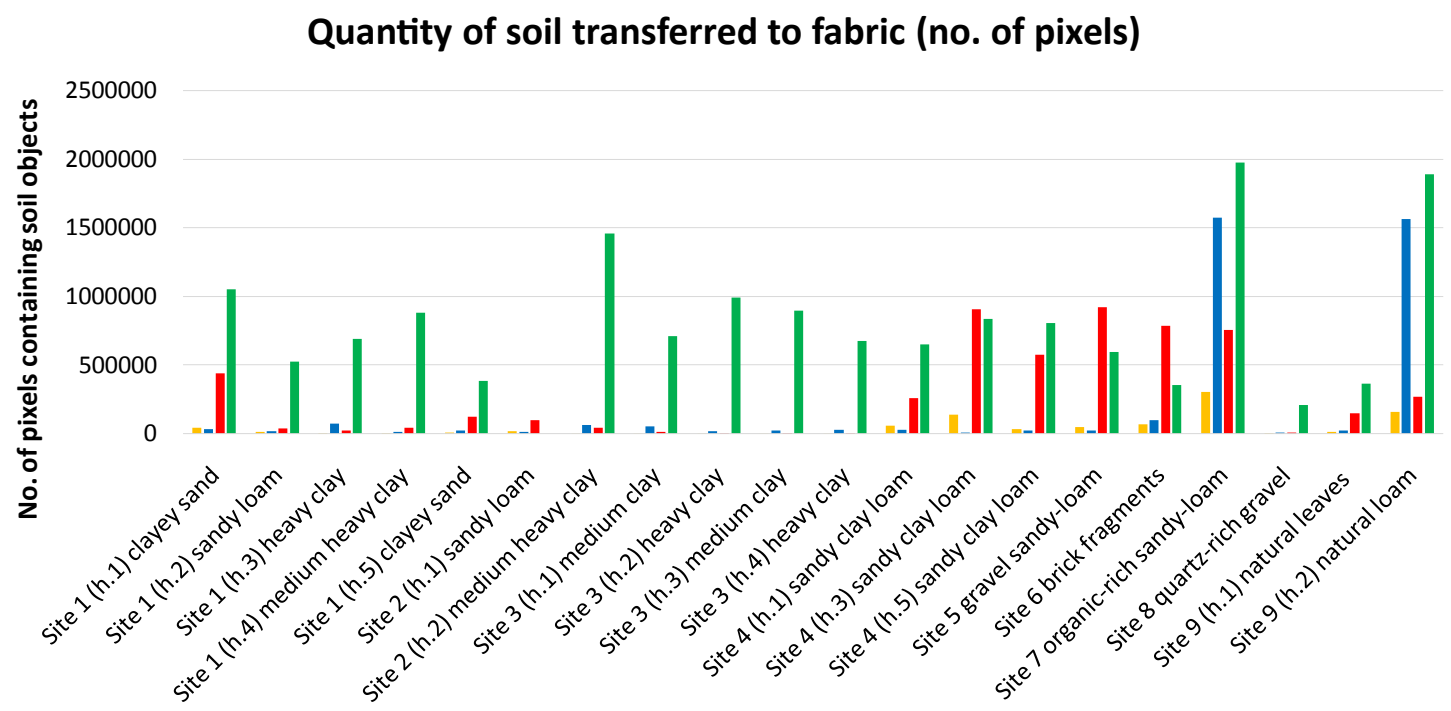

20 soil samples by location, soil type and method of soil transfer

$\square$ DRYPLACE $\quad \square$ WETPLACE $\quad$ DRYDRAG $\quad$ WETDRAG

Figure 11. Comparison of quantity of soil transferred to fabric using the soil transfer methods of placing and dragging weighted fabric across a soil surface. Quantity of trace soil transferred was analysed by number of pixels in digital photographs that contained individual or aggregate soil objects. Image processing numerical data was subdivided by location and soil moisture content [22].

\section{Conclusions}

In a controlled laboratory environment, 400 soil transference experiments (STEs) produced six transference patterns identified by naked eye and confirmed by simple light microscopy and image processing. The minimal quantities of trace soil transferred from only placing a simulated clothed body on a soil surface made it more difficult to discover a relationship between soil moisture, particle size, mineralogy and soil objects transferred. Dark organic loamy-sand textured soil transfer patterns enabled easiest recognition by naked eye against the white fabric. However, image processing was equally capable of recognising all soil objects, regardless of Munsell soil colour. With further software development, image processing could be programmed to identify trace soil objects on underlying fabrics of differing colours, patterns and textile weaves.

Image processing provided objective support to visual observations by showing:

(i) how the quantity of soil objects transferred is influenced by soil mineralogy and moisture content,

(ii) percentage of aggregate and individual soil objects transferred,

(iii) lack on soil object directionality when 'placing' is the soil transfer method,

(iv) the unique ranges of Munsell soil colours of trace soil on fabric and

(v) the negligible influence the four fabric types tested had on trace soil patterns produced.

\section{Acknowledgements}

We would like to thank the following people:
- The principal author's sister, Cheryl Robins, for sewing all the fabric squares used in the experiments.

- $\quad$ Associate Professor Ron Berry (Retired), CODES, School of Physical Sciences, The University of Tasmania, for providing invaluable technical expertise with operating image processing software.

- Richie Woolley, Technical Officer, Mineral Resources Tasmania, for providing mineralogical phase identification by manually comparing the measured XRD patterns with a series of similarly-prepared standards of the more common minerals to enable semi-quantitative XRD analysis.

- David Reid, Horticultural Coordinator, Royal Tasmanian Botanical Gardens, Hobart, for his invaluable historical knowledge regarding the establishment of the gardens and the heritage-listed convict-built Eardley Wilmot brick wall.

- Garth Oliver, Project Manager, Soil carbon, TIAR, School of Land and Food, University of Tasmania, for his technical support in the lab.

\section{References}

1. Fitzpatrick R, Raven M (2012) How pedology and mineralogy helped solve a double murder case: using forensics to inspire future generations of soil scientists. Soil Horiz 53: 14-29.

2. Fitzpatrick R, Raven M, Forrester S (2009) A systematic approach to soil forensics: criminal case studies involving transference from crime scene to forensic evidence. In: Ritz K, Dawson L, Miller D, editors. Criminal and Environmental Soil Forensics. Netherlands: Springer Netherlands p. 105-127.

3. Fitzpatrick R, Raven M (2013) Soils. In: Siegel J, Saukko P (eds.) Encyclopedia of Forensic Sciences. 2nded. Waltham, MA: Academic Press p. 206-212.

4. Fitzpatrick R (2013) Soil: forensic analysis. In: Jamieson A, Moenssens A (eds.) Wiley Encyclopedia of Forensic Science. Chichester, West Sussex.

5. Murray R (2011) Evidence from the Earth: Forensic Geology and Criminal Investigation. 2ndedn. Missoula, MT: Mountain Press Publishing. 
Murray KR (2017) An investigation of the pattern formed by soil transfer when clothing fabrics are placed on soil using visual examination and image processing analysis

6. Pye K (2007) Geological and Soil Evidence: Forensic Applications. Boca Raton, FL: CRC Press.

7. Ruffell A, McKinley J (2008) Geoforensics. Chichester: John Wiley \& Sons, Ltd.

8. Morgan R, Bull P (2007) Forensic geosciences and crime detection: Identification, interpretation and presentation in forensic geoscience. Minerva Medicolegale 127: 7389.

9. Bull PA, Parker A, Morgan RM (2006) The forensic analysis of soils and sediment taken from the cast of a footprint. Forensic Sci Int 162: 6-12. [Crossref]

10. Martin B (2012) The State of Western Australia v. Rayney. [No 3]: WASC. p. 1-369.

11. Martin B (2012) Judgement Summary: The State of Western Australia v. Rayney. [No 3]: WASC. p. 1-13.

12. Fitzpatrick R, Raven M, Self P (2011) Detailed mineralogical characterization of small brick and soil fragments $(<0.5 \mathrm{~mm}$ diameter $)$ by Synchrotron X-ray diffraction analyses for further forensic comparisons relating to operation Dargan. Centre for Australian Forensic Soil Science Restricted Client Report. p. 1-116.

13. Fitzpatrick R, Raven M (2012) The State of Western Australia v. Lloyd Patrick Rayney Trial: Request for Supplementary Statement Relating to Extraction of Particulate Material from Bra. Centre for Australian Forensic Soil Science. p. 1-17.

14. Raven M, Fitzpatrick R, Self P (2016) Trace evidence examination using laboratory and synchrotron X-ray diffraction techniques Paper no. 1131. 35th Internationa Geological Congress, Symposium on Forensic Soil Science and Geology. Cape Town, South Aftrica.

15. Murray K, Fitzpatrick R, Bottrill R, Berry R, Kobus H (2016) Soil transference patterns on bras: Image processing and laboratory dragging experiments. Forensic Sci Int 258 : 88-100.

16. Murray K, Fitzpatrick R, Bottrill R, Berry R, Woolley R, Doyle R, et al. (2015) Soil patterns on bra fabrics dragged across different soil surfaces using image analyses: laboratory dragging experiments. Acid Sulfate Soils Report No ASSC 096 Adelaide: The University of Adelaide, Australia. p. 1-100.

17. Murray K, Fitzpatrick R, Bottrill R, Kobus H (2017) Patterns produced when soil is transferred to bras by placing and dragging actions: the application of digital photography and image processing to support visible observations: [In press]
18. Isbell R, National_Committee_on_Soils_and_Terrain (2016) The Australian Soil Classification. 2ndedn. Clayton, Victoria, Australia: CSIRO Publishing.

19. IUSS Working Group WRB (2014) World Reference Base for Soil Resources 2014 International soil classification system for naming soils and creating legends for soil maps. World Soil Resources Reports No 106. Rome: FAO. p. 1-181.

20. Soil Survey Staff (2014) Keys to Soil Taxonomy. 12thedn. Washington, DC: United States Department of Agriculture, Natural Resources Conservation Service.

21. Galbraith J (2012) Rationale for proposed changes to Soil Taxonomy concerning the International Committee for Anthropogenic Soils. Soil Horizons 2-6.

22. Murray K, Fitzpatrick R, Bottrill R, Kobus H (2016) Soil transference patterns on clothing fabrics and plastic buttons: image processing and laboratory dragging experiments. Forensic Science and Criminology 2(1): 1-12

23. Department of Primary Industries P, Water and Environment (2015) LISTmap - Land Information System Tasmania. Tasmania: DPIPWE. http://maps.thelist.tas.gov.au/ listmap/app/list/map.

24. Fitzpatrick R (2013) Demands on soil classification and soil survey strategies: specialpurpose soil classification systems for local practical use. In: Taha F, Abdelfattah M (eds.) Developments in Soil Classification, Land Use Planning and Policy Implications: Innovative Thinking of Soil Inventory for Land Use Planning and Management of Land Resources. Netherlands: Springer. p. 51-83.

25. Jantzi S, Almirall J (2011) Characterization and forensic analysis of soil samples using laser-induced breakdown spectroscopy (LIBS). Anal Bioanal Chem 400: 3341-3351. [Crossref]

26. Marumo Y (2003) Forensic examination of soil evidence. National Research Institute of Police Science 7: 95-111.

27. Sugita R, Marumo Y (1996) Validity of color examination for forensic soil identification. Forensic Science Int 83: 201-210.

28. Holcombe R (2011) GEOrient. Queensland, Australia: School of Earth Sciences, University of Queensland.

29. Munsell_Color_Company. Munsell Soil Color (2009) Book. Michigan: Munsell Color $\mathrm{X}$-rite.

30. McDonald R, Isbell R (2009) Soil Profile. 3rdedn. Melbourne: CSIRO Publishing.

Copyright: ( 2017 Murray KR. This is an open-access article distributed under the terms of the Creative Commons Attribution License, which permits unrestricted use, distribution, and reproduction in any medium, provided the original author and source are credited. 